\title{
SPONTANEOUS RUPTURE OF THE HEART IN THE NEWBORN INFANT
}

\author{
BY \\ W. E. HUNT \\ From the Bernhard Baron Memorial Research Laboratories, Queen Charlotte's Maternity Hospital, London
}

(RECEIVED FOR PUBLICATION OCTOBER 5, 1951)

The pericardial sac of the newborn infant is frequently found at necropsy to contain a small quantity of thin, serous fluid, usually greenish; in the full-term infant this may amount to as much as 3 or $4 \mathrm{ml}$. When, in an attempt at resuscitation, an intracardiac injection has been made shortly before death, this pericardial effusion may be tinged with a variable amount of blood, and the site of entry of the needle into the heart is usually clearly visible as a tiny area of subpericardial haemorrhage on the ventricular wall. Such a slight degree of traumatic haemopericardium is seldom of any significance, since the myocardium closes over the needle track and effectively seals it so that no more blood can be expressed from the site of injection. On the other hand, haemopericardium due to spontaneous rupture of the heart is a very rare condition in the newborn, and the following case, in which the lesion occurred shortly before delivery, is presented as an example of an unusual cause of stillbirth.

\section{Case Report}

The mother, a primigravida, was aged 28 years; blood group A Rh-positive. Her Wassermann reaction was negative. She had a long history of varicose veins with ulceration of the right leg, and four years before this pregnancy she had been admitted to hospital for a Trendelenberg's operation, and again, in the following year, for ligation and excision of the veins below the knee. Otherwise her past history was uneventful. Both her parents died in old age, her father of cardiovascular disease, and her mother of 'senile degeneration'; she had no brothers or sisters. Her husband was a twin. The pregnancy was normal except that the foetus presented as a breech, and at the thirty-sixth week of pregnancy she was admitted for external cephalic version under sedation. The foetal heart rate both before and after the version, which was unsuccessful, was 140 per minute. Two weeks later the membranes ruptured spontaneously and the patient was admitted in labour, with the cervix fully dilated. The first and second stages of labour lasted three and a quarter hours and resulted in the delivery of a stillborn foetus as an assisted breech under gas, oxygen, and trilene anaesthesia. Twenty-five minutes before delivery there was some doubt whether the foetal heart could be heard, but it was not heard immediately before delivery, nor was there any pulsation in the cord when the foetus had been delivered as far as the umbilicus. No intracardiac injection was given after delivery. The third stage of labour, which lasted three hours, was complicated by a retained placenta, which required manual removal under gas, oxygen, trilene and ether anaesthesia. It was then discovered that the mother had a bicornuate uterus, and the placenta, which was not morbidly adherent, was present in the right horn. One pint of group A Rh-positive blood was given, after which the puerperium was uneventful.

Macroscopic Examination. The body was that of a fresh female foetus of approximately 38 weeks maturity. The weight was $2.58 \mathrm{~kg}$., and the length, crown-rump $34 \mathrm{~cm}$., crown-heel $49 \mathrm{~cm}$. There were no external features of note, and no cyanosis or oedema.

The thoracic contents were exposed by incising the costal cartilages at the lateral ends, and removing the sternum by blunt dissection to avoid injury to the underlying structures. The pericardial sac was found to be greatly distended by a large amount of fresh fluid blood. When this had been removed, and the heart had been gently wiped clean, a slow ooze of blood was seen issuing from a point on the anterior aspect of the right atrium, close to the root of the atrial appendix; examination of this area under 30 magnification revealed a tiny round perforation in the atrial wall, less than $0.5 \mathrm{~mm}$. in diameter. The right atrium was moderately distended; the other chambers, all of which contained fluid blood, appeared normal in size. No subpericardial haemorrhages were found. All the cusps of both the mitral and tricuspid valves showed on the internal surfaces numerous rounded haematomata of varying sizes, the largest being approximately $1 \mathrm{~mm}$. in diameter. The aortic and pulmonary valves and the great vessels were normal, and the ductus arteriosus was patent. The coronary vessels, which followed their normal course, were not engorged, and the myocardium showed no unusual features apart from the perforation in the right atrium.

There was no fluid in the pleural sacs. The trachea was clear, but both bronchi were completely occluded by a mass of thick mucus. The lungs were uniformly plum-coloured, with a few subpleural petechiae, and they showed no evidence of aeration; both lungs, and all portions from them, sank in water.

The stomach, intestines, liver (135 g.), spleen (8 g.) and pancreas were normal. There was a small spherical 
accessory spleen, $5 \mathrm{~mm}$. in diameter, lying in the gastrosplenic ligament. The right kidney lay at a more caudal level than the left, so that it was completely separated from the right suprarenal gland. In both kidneys there was much haemorrhage surrounding the pelvis and calyces, but the cut surface showed a normal renal pattern. Since it did not lie in contact with the kidney, the right suprarenal showed no evidence of moulding on its inferior surface, but had assumed the shape of a flattened, oval disc, with diameters of approximately $2 \times 4.5 \mathrm{~cm}$.: the left suprarenal was normal. The head showed a slight subcutaneous haemorrhage near the anterior fontanelle, but there was no intracranial haemorrhage, and the dural membranes were undamaged. The brain showed considerable congestion, both externally and internally, but was otherwise normal.

Microscopic Examination. Serial sections were cut in the coronal plane through the anterior wall of the right atrium in the region of the perforation. Of these sections, that illustrated in Fig. 1 shows the perforation at its widest part; it appeared as an interruption in the

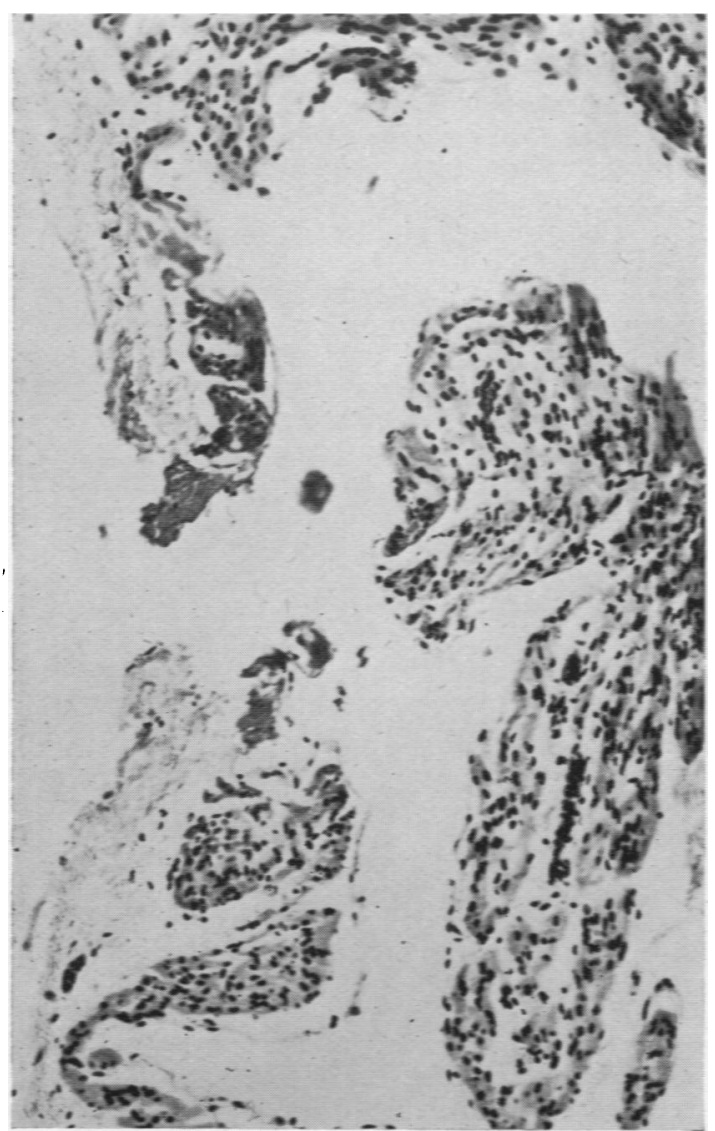

Fig. 1.-Photomicrograph of part of the anterior wall of the right atrium showing the site of rupture, and the hyaline degeneration of the myocardium on each side of the gap in the atrial wall. Haematoxylin and cosin $(\times 150)$. continuity of the atrial wall, measuring approximately $140 \mu$ between the torn edges of the visceral pericardium. On each side of the rupture there was a small mass of acellular hyaline material, which gave a strongly acidophilic reaction with haematoxylin and eosin and with carbol fuchsin, and the rupture appeared to have occurred through this area of hyaline degeneration; the myocardium on the immediate right-hand side of the rupture was affected by a similar change, but the degeneration in these fibres was not as far advanced as at the site of the rupture. There was no evidence in this, or in any of the serial sections, of any cellular reaction or fibrosis in the region of the perforation, and the neighbouring capillaries were normal and showed no obstruction or occlusion. The lesion did not resemble an infarct, but rather appeared to be a small zone of hyaline degeneration occurring in the myocardium at a point where the latter was very thin. Although there were several other places in the atrial wall where the myocardium was reduced to a thickness of not more than $\mathbf{2}$ or $\mathbf{3}$ cells (one such place is included near the lower left-hand edge of Fig. 1) no other evidence of hyaline change was seen in the atrial wall, the myocardium of which appeared entirely normal.

The only other feature of note in the heart was seen in a section from the anterior wall of the right ventricle, which included part of the anterior cusp of the tricuspid valve. Within the latter were three large, round, circumscribed haematomata, stuffed with erythrocytes, and showing no evidence of coagulation. Since such collections of blood in the valve cusps are a fairly common finding in the heart of the newborn infant, these lesions were not in any way related to the degeneration of the atrial wall. The ventricular myocardium and the coronary vessels were normal.

\section{Discussion}

The great majority of cases of spontaneous rupture of the heart occur in old persons, the incidence being highest in the seventh and eighth decades. Diamond (1945) found that the average age of patients dying with rupture of the ventricle was 66 years, and in their analysis of 602 cases, which included three previously reported series, Krumbhaar and Crowell (1925) found only four occurring in the first decade, but they did not give the ages of these patients. Lyon (1950) states that haemorrhage into the pericardial sac can occur in the neonatal period as a result of birth trauma, and rarely in cases of blood dyscrasia, but he does not mention spontaneous rupture of the heart as a cause of haemopericardium in infancy.

Acute spontaneous haemopericardium in the adult may arise from a number of lesions, of which rupture of a myocardial infarct is the commonest (Fulton, 1940). Myocardial infarction has been found in patients of all ages, and is known to occur in infancy. In a survey of the literature Ravich 
and Rosenblatt (1947) were able to collect four reported cases, the ages of which ranged from 7 weeks to 11 months, and they added two further examples in infants dying at 2 days and $10 \frac{1}{2}$ hours respectively, thus showing that the lesion could develop during intra-uterine life. Another instance was reported by Knop and Bennett (1944): in this infant, who died at the age of 5 days, the lesion was associated with congenital absence of the left coronary artery and an inadequate left branch from the right coronary.

None of these cases, however, was complicated by rupture of the heart, and the only recorded example of such a lesion in the newborn appears to be that of Benmussa, Caruana and Hamza (1950) in an infant who died at the age of 24 days. The child, a boy, was admitted to hospital on the seventeenth day with diarrhoea and failure to gain weight. He was treated with subcutaneous fluids, penicillin and sulphadiazine, but developed signs of pneumonia and died suddenly during an intense cyanotic attack seven days after admission. At necropsy the mediastinum was found to be full of blood, and there was a laceration of the parietal pericardium extending over the whole of its anterior surface; the haemorrhage had arisen from a horizontal rupture of the right ventricle, $2 \mathrm{~cm}$. in length. The coronary arteries appeared normal on naked-eye examination, and, apart from a patent ductus arteriosus, there were no other lesions in the heart or great vessels. There was also evidence of acute enteritis and bronchopneumonia. Microscopically there was capillary stasis, with some small haemorrhages and phagocytosis of pigment granules, in the region of the rupture, but, for the most part, the myocardial fibres showed no degeneration except at one point. Unfortunately, the coronary arteries were not examined microscopically, and the authors could find no cause for the haemorrhagic infarction that had resulted in the rupture of the right ventricle; they suggest, however, that certain factors may have contributed to the formation of the rupture. Thus, 24 hours before death, the infant was inadvertently given as much as $150 \mathrm{ml}$. of plasma intravenously in only 15 minutes, and this sudden overloading of the circulation may have started a tear in the ventricular wall, already weakened by an infarct; the normal contractions of the ventricle may then have extended this tear from within outwards, and the asphyxia due to the concomitant bronchopneumonia may have constituted a third factor, which acted by causing dilatation of the right ventricle and thinning of its wall.

In the case presented here the very small size of the perforation in the right atrium made it unlikely that the degeneration of the atrial myocardium was due to infarction. Moreover, in serial sections taken through the tissue on each side of the perforation, no evidence of occlusion or obstruction could be found in the neighbouring blood vessels, nor was there any evidence of haemorrhage or fibrosis in the vicinity of the rupture. The lesion appeared to be a focal hyaline degeneration of the myocardium, the cause of which is not clear. The atrial wall in the newborn infant is so thin that it becomes transparent when the atrium is greatly dilated, and the external surface then appears as a patchwork of dark blue areas separated by the brownish striae of the thicker muscular bundles. In this case myocardial degeneration had occurred at a point where there was only a very thin layer of muscle, so that a weakened area, unable to withstand any increase in the intra-atrial blood pressure, was formed. The presence of subpleural petechiae on the lungs and the considerable degree of cerebral vascular congestion indicate that the foetus was subjected to some degree of anoxia during labour, the origin of which may possibly have been related to the abnormal location of the placenta in one horn of the bicornuate uterus, or to the breech delivery of the foetus.

Since the birth of this stillborn child the mother has had a second living child which, on account of the bicornuate uterus, was delivered at 37 weeks by Caesarean section.

\section{Summary}

A case is reported of a stillbirth, in which foetal death was caused by spontaneous perforation of the right atrium with subsequent haemopericardium, shortly before delivery. The rupture occurred at a point where the atrial myocardium had undergone focal hyaline degeneration, no cause for which was found.

This is believed to be the second recorded case of spontaneous haemopericardium occurring in the newborn infant, and the first in which this lesion has been the cause of stillbirth.

I wish to thank Professors James Young and J. H. Dible, for their permission to publish the details of the case, and Dr. J. Murray, for his criticism and advice. My thanks are also due to Mr. E. Clark, F.I.M.L.T., for the photomicrograph.

\section{References} Benmussa, S.. Caruana, M. and Hamza. B. (1950). Arch. franc.
Pédiat.. 7, 607.

Diamond, J. L' (1945). Lancet, 65, 396.

Fulton, M. N. (1940). Med. Clin. N. Amer, 24, 1371

Knop, C. Q. and Bennett, W. A. (1944). Proc. Mayo Clin., 19, 574 Knop, C. Q. and Bennett, W. A. (1944). Proc. Mayo Clin., 19, 574.
Krumbhar, E. B. and Crowell, C. (1925). Amer. J. med. Sci., 17, 828.

Lyon, R. A. (1950). In ' Mitchell-Nelson Textbook of Pediatrics', 5th ed. p. 1070 . Phitadelphia.

Ravich, R. M. and Rosenblatt, P. (1947). J. Pediat., 31, 266. 